\title{
Determination of the optimal color space from eight types of color spaces for distinguishing small retinal hemorrhages from dust artifacts
}

\author{
Naoto Suzuki \\ Dept. of Medical Science and Technology, Hiroshima International University, Higashi-hiroshima, Japan \\ Email address: \\ n-suzuki@hs.hirokoku-u.ac.jp
}

\section{To cite this article:}

Naoto Suzuki. Determination of the Optimal Color Space from Eight Types of Color Spaces for Distinguishing Small Retinal Hemorrhages from Dust Artifacts. International Journal of Biomedical Science and Engineering, Vol. 1, No. 1, 2013, pp. 10-19. doi: $10.11648 /$ j.ijbse.20130101.12

\begin{abstract}
To determine the optimal color space from eight types of color spaces for distinguishing small retinal hemorrhages from dust artifacts in cases of early diabetic retinopathy. We constructed an experimental device, which comprised an illumination optical system and a photographic optical system separated by a mirror having a hole. This device included a canon EOS 50D camera, an EF $50 \mathrm{~mm}$ f/1.8-2 camera lens, a Speedlite 270EX flash, an object lens, four double-convex lenses, three aperture stops, and six artificial eyes. The hemispherical eye ground was made of polythene terephthalate, which was painted with six matt color sprays: red, coffee, ocher, yellow, ivory, and orange. Five fragments of house dust on the object lens and the two lenses were photographed under each artificial eye. The RGB color space, measured by Paint Shop Pro from pictures, was changed into seven types of color spaces: XYZ, CMY, HSL, HSV, HSI, $\mathrm{L}^{*} \mathrm{a}^{*} \mathrm{~b}^{*}$, and $\mathrm{L}^{*} \mathrm{u}^{*} \mathrm{v}$. The $\mathrm{L}^{*} \mathrm{u}^{*} \mathrm{v} *$ color space was the optimal one as it demonstrated the highest sensitivity and the best reproducibility. This result demonstrated that this color space could distinguish small hemorrhages from dust artifacts. Next, we analyzed the $\mathrm{L}^{*} \mathrm{u}^{*} \mathrm{v}$ color space and compared the following three types of house dust positions: "on an object lens," "on a photographic optical system," and "on an illumination optical system." The house dust position "on an object lens" had the highest sensitivity and the best reproducibility. However, the positions "on a photographic optical system" and "on an illumination optical system" had high sensitivity and good reproducibility only under certain conditions. In addition, no differences were found among the six types of fundus colors.
\end{abstract}

Keywords: Color spaces, Diabetic Retinopathy, Dust Artifacts, Small Retinal Hemorrhages

\section{Introduction}

In 1999, researchers in the United States estimated that the number of American patients with diabetes would range from 16 to 30 million over the next 30 years [1]. In comparison, the number of Japanese patients with diabetes, including potential patients, reached 16.2 million in 2005 [2]. Many patients with diabetic retinopathy require regular ophthalmological examinations to prevent loss of eyesight [3-6]. For this reason, early diagnosis is very important; however, many retinal hemorrhages are minuscule [7 - 9]. Detection of small retinal hemorrhages in patients with cloudy ocular media, such as in a cataract, is especially challenging. Moreover, as shown in Fig. 1, magnified images taken with a fundus camera can be unclear.

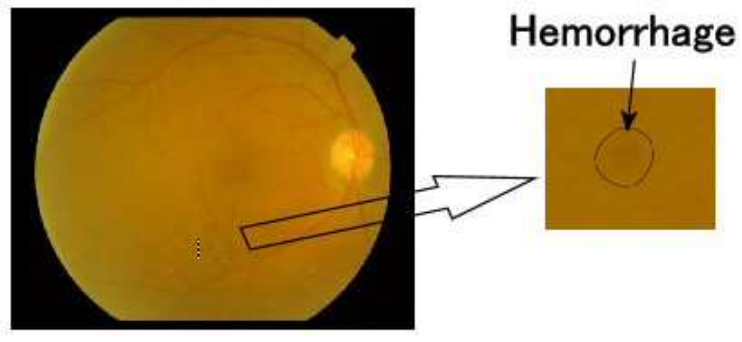

Figure 1. The area of small retinal hemorrhage in the fundus image of patient with diabetic retinopathy

White spots may appear in a photograph because of house dust particles that are present in the air of the room, in which the photograph has been taken [10 - 11]. Black spots may appear in a photograph, if dust particles are 
attached to the lens of the camera. These white and black spots are called dust artifacts [12 - 14].

The components of a fundus camera include the camera, strobe, object lens, other lenses, and mirror with a hole, another mirror, some aperture stops, and more parts [15 16]. If dust particles adhere to any of these optical components, dust artifacts will be visible on the fundus images. Previously, the following two methods of image clarification were explored: (1) deleting dust artifacts from images and (2) removing house dust particles from parts of the device.

However, these researches did not explore the use of the fundus camera in medical diagnoses [17 - 21]. Methods of image processing and deleting image artifacts other than dust artifacts have been explored. Moreover, image artifacts have been extracted from the existing images [22 - 25]. We conducted experiments to determine whether small retinal hemorrhages due to diabetic retinopathy could be distinguished from dust artifacts by using the concept of color space [26].

The most important element for the concept of color space is lightness. House dust is complicated in form and uneven in density. The quantity of light is irregular because of diffused reflection. Dust artifacts create a spotty pattern. In diabetic retinopathy, the fundus is reddish-brown or yellow in color. Therefore, in this study, red, coffee, ocher, yellow, orange, and ivory colors were investigated.

The CIE and Munsell color systems can be used to define colors in a color space [25]. The CIE color system is characterized by a number of color spaces, including red, green, and blue (RGB); cyan, magenta, and yellow (CMY); $\mathrm{XYZ}$; L*a*b*; and $\mathrm{L}^{*} \mathrm{u}^{*} \mathrm{v}^{*}$ [25]. In the XYZ color space, $\mathrm{Y}$ expresses lightness, $\mathrm{Z}$ expresses degree of blueness, and $\mathrm{X}$ expresses other elements. In the $\mathrm{L}^{*} \mathrm{a}{ }^{*} \mathrm{~b}^{*}$ color space, " $\mathrm{L} *$ " expresses lightness while both " $a *$ " and " $b$ " represent complementary colors. " $a *$ " represents the color between red, magenta and green, while " $b$ " represents the color between yellow and blue. In the $\mathrm{L}^{*} \mathrm{u}^{*} \mathrm{v}^{*}$ color space, " $\mathrm{L} *$ " expresses lightness while both " $\mathrm{u}$ " " and " $\mathrm{v} *$ " are chromaticity coordinates. The Munsell color system specifies colors on the basis of hue, saturation, and lightness (HSL); hue, saturation, and value (HSV); and hue, saturation, and intensity (HSI) [27 - 29].

\section{Purpose}

1. In order to distinguish small diabetic retinal hemorrhages from dust artifacts, we built an experimental device with an optical system that was similar to that of a fundus camera. The device was designed to photograph the fundus of artificial eyes, for which six of the following fundus colors were used: red, coffee, ocher, yellow, orange, and ivory.

2. The experimental device photographed the dust artifacts of the six artificial eyes, and fundus images of five patients with diabetic retinopathy were taken.

3. Paint Shop Pro measured the RGB color spaces of both the small hemorrhages and dust artifacts.
The RGB color space was transformed into the following seven kinds of color spaces: XYZ, CMY, HSL, HSV, HSI, $\mathrm{L}^{*} \mathrm{a} * \mathrm{~b} *$, and $\mathrm{L}^{*} \mathrm{u}^{*} \mathrm{v}^{*}$. We analyzed eight types of color spaces and determined the highly sensitive color space.

\section{Methods}

\subsection{Distinguishing Small Retinal Hemorrhages from Dust Artifacts Using Eight Types of Color spaces}

Fig. 2: (left) demonstrates the division of the fundus image into hemorrhagic area and perihemorrhagic area. Paint Shop Pro v. 8.0 was used to visualize RGB color spaces of both these areas.

Equations:

(1) demonstrates the average RGB color space of the hemorrhagic area, (aveRGBhm)

(2) demonstrates the average RGB color space of the perihemorrhagic area (aveRGBph).

$$
\begin{aligned}
\text { aveRGBhm } & =\left[\begin{array}{l}
\text { AveR_hm } \\
\text { AveG_hm } \\
\text { AveB_hm }
\end{array}\right] \\
\text { aveRGBph } & =\left[\begin{array}{l}
A v e R_{-} p h \\
A v e G_{-} p h \\
A v e B_{-} p h
\end{array}\right]
\end{aligned}
$$

Fig. 2 (right): demonstrates an image of the dust artifact and the periartifact area. Paint Shop Pro v. 8.0 was used to visualize RGB color spaces of both these areas. RGB values are calculated as a minimum of 0 and a maximum of 255. These RGB values were transformed into a minimum of 0 and a maximum of 1 by dividing them by 255 .
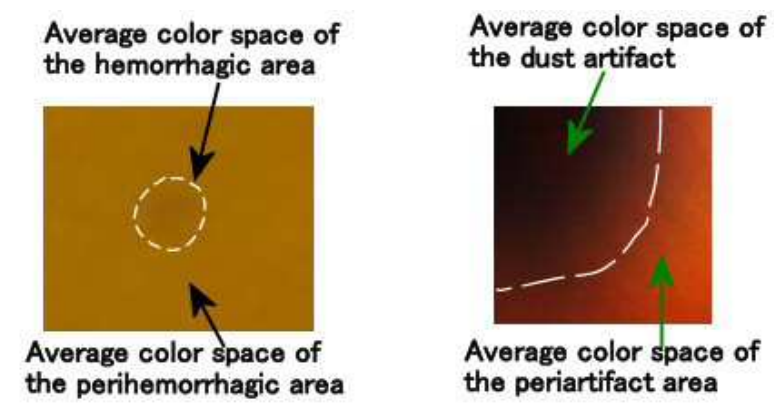

Figure 2. The average color space of both the hemorrhagic and perihemorrhagic area (left), and the average color space of the dust artifact and the periartifact area (right).

Equations:

(3) demonstrates the average RGB color space of the dust artifact (aveRGBda)

(4) demonstrates the average RGB color space of the periartifact area (aveRGBpa).

$$
\text { aveRGBda }=\left[\begin{array}{l}
A v e R_{-} d a \\
A v e G_{-} d a \\
A v e B_{-} d a
\end{array}\right]
$$




$$
\text { aveRGBpa }=\left[\begin{array}{l}
A v e R_{-} p a \\
A v e G_{-} p a \\
A v e B_{-} p a
\end{array}\right]
$$

Equations:

(5) demonstrates the average color space of the hemorrhagic area (avehm)

(6) demonstrates the average color space of the perihemorrhagic area (aveph)

(7) demonstrates the average color space of the dust artifact (aveda), and

(8) demonstrates the average color space of the periartifact area (avepa).

$$
\begin{gathered}
\text { avehm }=\left[\begin{array}{l}
\text { Ave1_hm } \\
\text { Ave2_hm } \\
\text { Ave3_hm }
\end{array}\right] \\
\text { aveph }=\left[\begin{array}{l}
\text { Ave1_ph } \\
\text { Ave2_ph } \\
\text { Ave3_ph }
\end{array}\right] \\
\text { aveda }=\left[\begin{array}{l}
\text { Ave1_da } \\
\text { Ave2_da } \\
\text { Ave3_da }
\end{array}\right] \\
\text { avepa }= \\
{\left[\begin{array}{l}
\text { Ave1_pa } \\
\text { Ave2_pa } \\
\text { Ave3_pa }
\end{array}\right]}
\end{gathered}
$$

Each ingredient $(1,2,3)$ of the color spaces demonstrates (R, G, B); (X, Y, Z); (C, M, Y); (H, S, L); (H, S, V); (H, S, I); (L*, $\left.\mathrm{a}^{*}, \mathrm{~b}^{*}\right)$; and $\left(\mathrm{L}^{*}, \mathrm{u}^{*}, \mathrm{v}^{*}\right)$. The RGB color space was transformed into seven types of color spaces: CMY, HSL, HSV, HSI, L*a*b*, L*u*v* [30 - 31] and, XYZ [31].

Equations:

(9) demonstrates the change in the color space of the hemorrhagic area (CngHm)

(10) demonstrates the color space change in the dust artifact (CngDa).

Each ingredient of $\mathrm{CngHm}$ is characterized by the ratio of change in the hemorrhagic area to that in the perihemorrhagic area. Each ingredient of $\mathrm{CngDa}$ is characterized by the ratio of change in the dust artifact to that in the periartifact area.

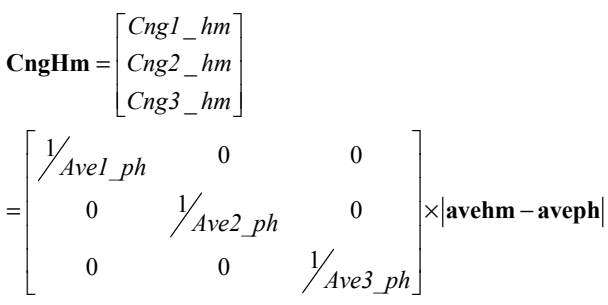

$$
\begin{aligned}
& \text { CngDa }=\left[\begin{array}{l}
\text { Cng1_da } \\
\text { Cng2_da } \\
\text { Cng3_da }
\end{array}\right] \\
& =\left[\begin{array}{ccc}
1 / \text { Ave1_pa } & 0 & 0 \\
0 & 1 / \text { Ave2_pa } & 0 \\
0 & 0 & 1 / \text { Ave3 } p a
\end{array}\right] \times \mid \text { aveda }- \text { avepa } \mid
\end{aligned}
$$

In general, hue is expressed using a $360^{\circ}$ color circle [29], [32 - 33]. Therefore, in the case of hue, equations (11) and (12) are used.

$$
\begin{aligned}
& 1 / \text { Ave1_ph }=1 \\
& 1 / \text { Ave1_pa }=1
\end{aligned}
$$

Equations:

(13) demonstrates AveCngHm, which is the average of CngHm.

(14) demonstrates the color space for evaluating Ev, which is the ratio of change of CngDa to AveCngHm.

$$
\text { AveCngHm }=\left[\begin{array}{l}
\text { AveCng1_hm } \\
\text { AveCng2_hm } \\
\text { AveCng3_hm }
\end{array}\right]
$$

$\mathbf{E v}=\left[\begin{array}{c}E v 1 \\ E v 2 \\ E v 3\end{array}\right]$

$=\left[\begin{array}{ccc}1 / \text { AveCng1_hm } & 0 & 0 \\ 0 & 1 / \text { AveCng2_hm } & 0 \\ 0 & 0 & 1 / \text { AveCng3_hm }\end{array}\right] \times \mathbf{C n g D a}$

\subsection{Fundus Photographs of Diabetic Retinopathy}

Fundus photographs were taken in five patients using the fundus camera Topcon TRC-50EX mydriatic retinal camera with a Nikon digital camera D1x. The image sensor was a $23.7 \times 15.6-\mathrm{mm}, 12$-bit RGB CCD [34]. The file format is JPEG baseline-compliant. The number of recorded pixels is $2000 \times 1312$. Paint Shop Pro v. 8.0 was used to visualize the average RGB color spaces in two locations on the photograph: the hemorrhagic area and the perihemorrhagic area.

\subsection{Experimental Device}

The experimental device, shown in Fig. 3, was equipped with an illumination optical system and a photographic optical system separated by a mirror with a hole $4 \mathrm{~mm}$ in diameter. The device consists of a canon EOS 50D camera with an EF 50-mm f/1.8-2 camera lens, a Speedlite 270EX flash, an object lens with $50-\mathrm{mm}$ focal length and a center thickness of $16 \mathrm{~mm}$, four double-convex lenses with focal lengths of $100 \mathrm{~mm}$ and center thicknesses of $10 \mathrm{~mm}$, three 
aperture stops, a mirror with a hole $4 \mathrm{~mm}$ in diameter, another mirror and six artificial eyes.

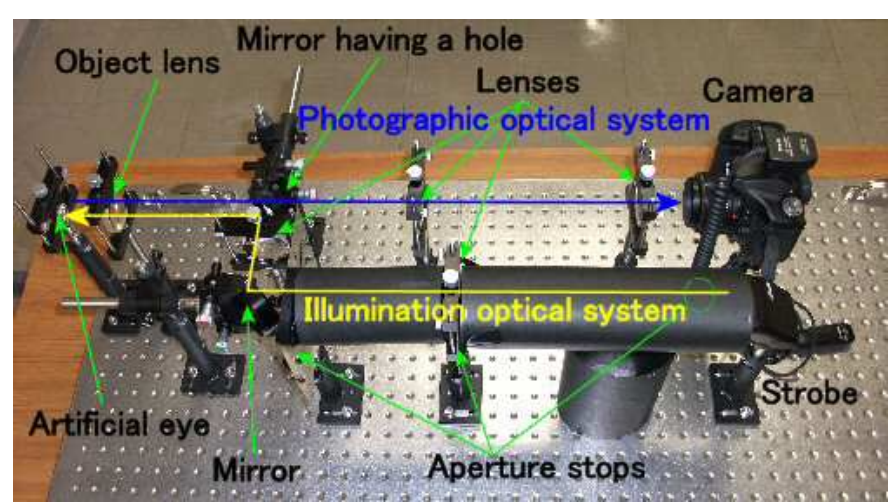

Figure 3. The experimental device

The object lens, four double-convex lenses and two mirrors are all $50 \mathrm{~mm}$ in diameter. An MgF2 coating was applied to the surface of all lenses. The image sensor used was a $22.3 \times 14.9-\mathrm{mm}$ CMOS sensor [35]. The file format was JPEG, RAW (14-bit Canon original). The number of recorded pixels was $4752 \times 3168$. Fig. 3 shows the aperture stop equipped with a $13-\mathrm{mm}$-diameter hole on the right side of the device. The hole in the middle aperture stop was 45 $\mathrm{mm}$ in diameter. The aperture stop on the left side of the device was equipped with a 39-mm-diameter hole. Three wires hang a black plate $15 \mathrm{~mm}$ in diameter in the center of the hole.

\subsection{Optical System Diagram of the Experimental Device}

The optical system of the experimental device was designed using optical design software (OpTaliX-LT 7.11). Fig. 4 shows an illumination optical system and a photographic optical system, including the distance between lenses and the distance between a lens and a mirror per $\mathrm{mm}$. The axial distance from eyeground to the image surface was $797.3 \mathrm{~mm}$ and that from eyeground to the strobe surface was $858.9 \mathrm{~mm}$.

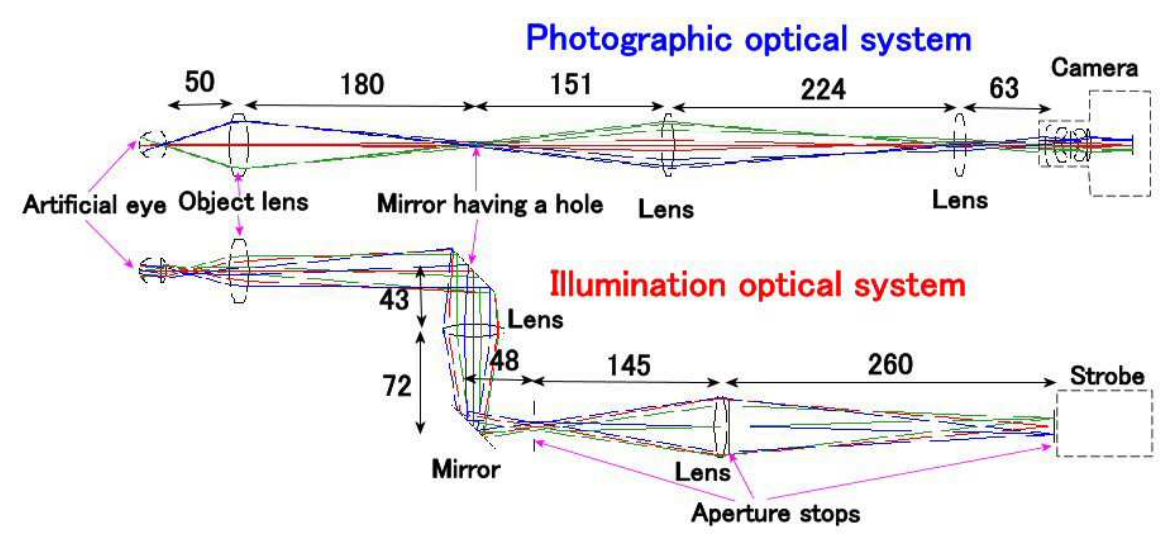

Figure 4. Optical system diagram of the experimental device

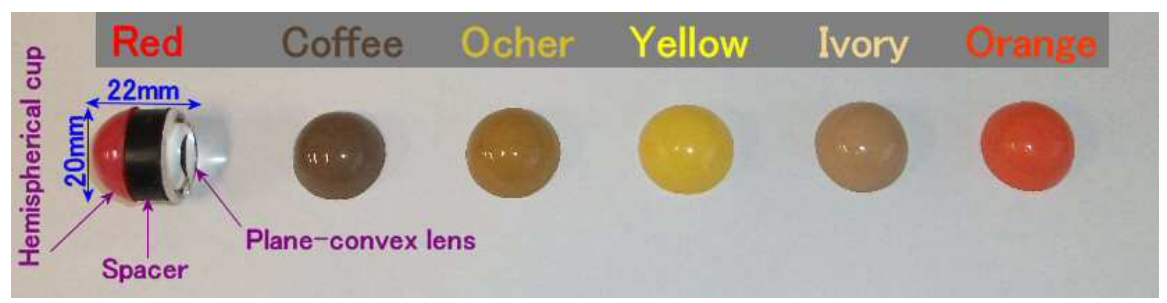

Figure 5. Artificial eyes

\subsection{Artificial Eyes}

The artificial eye consists of a plane-convex lens, a black spacer with a hole $18 \mathrm{~mm}$ in diameter and a hemispherical cup. The plane-convex lens was $20 \mathrm{~mm}$ in diameter, with a 4.6-mm center thickness and a $17.4-\mathrm{mm}$ back focal length. The hemispherical cup was $20 \mathrm{~mm}$ in diameter with a thickness of $0.5 \mathrm{~mm}$. The distance from the surface of the plane-convex lens to the eyeground was $22 \mathrm{~mm}$. MgF2 coating was applied to the surface of the plane-convex lens. The hemispherical cup was made of polyethylene terephthalate and painted using four matt color sprays (Asahipen Corp., Osaka, Japan): red, coffee, ocher, and yellow (Fig. 5). "Ruby red" was used as red, "Mexican sand" was used as coffee, "gold amber" was used as ocher, "canary yellow" was used as yellow, "light khaki" was used as ivory, and "sun flower" was used as orange.

\subsection{Specimens}

We prepared five types of fragments of house dust measuring about $5 \times 5 \times 5 \mathrm{~mm}^{3}$. Each fragment was set at points P1-P6 on a lens. Then fragments at each point were photographed one by one, as shown in Fig. 6. Paint Shop Pro v. 8.0 was used to visualize the RGB color space of four areas, as shown in Fig. 2. 


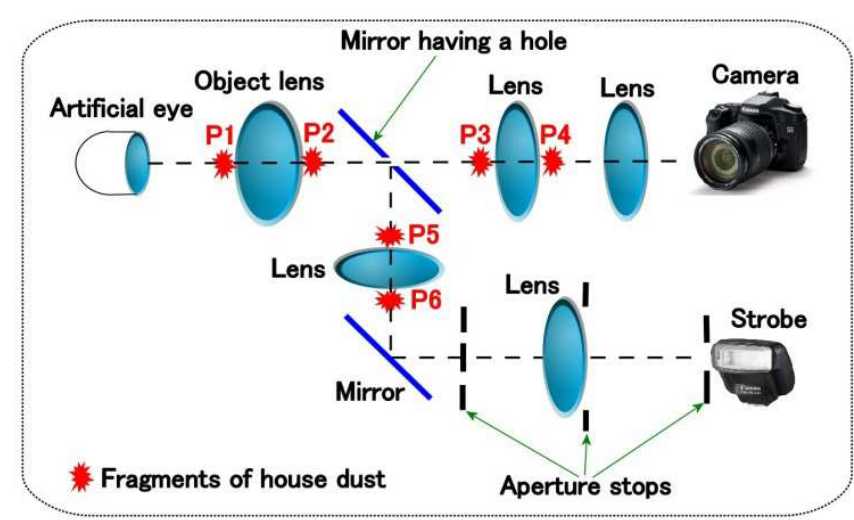

Figure 6. Fragments of house dust were set on the lens at points P1-P6

\subsection{Calculation of Evaluation Space for House Dust Using Eight Kinds of Color Spaces}

Equation (14) shows the color space for evaluation (Ev), which is the ratio of change of CngDa to AveCngHm. $\mathrm{CngDa}$ is the color space change in the dust artifact and AveCngHm is the average of the color space change in the hemorrhagic area. The greater the absolute values of Ev1, Ev2, and Ev3, the greater the extent to which the color space can be used to distinguish small hemorrhages from dust artifacts. These absolute values must be $>1.0$.

\section{Results}

\subsection{Transformed Color Spaces of the Hemorrhagic Area}

Fig. 7: Ten images of small retinal hemorrhagic areas from five fundus images. Paint Shop Pro v. 8.0 was used to visualize both aveRGBhm and aveRGBph in the images. The transformed color space of the hemorrhagic areas is CngHm. CngHm was calculated by substituting aveRGBhm and aveRGBph in equations (5), (6), (9), and (11). Table 1 shows the averages and standard deviations of CngHm in the eight types of color spaces.
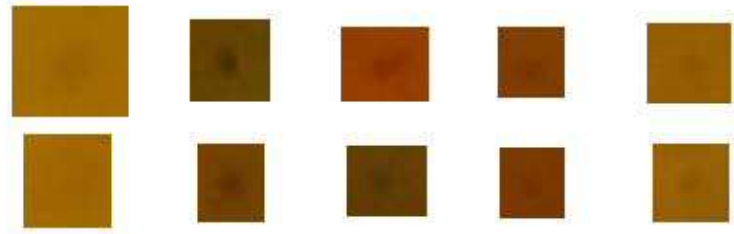

Figure 7. Ten images of the small hemorrhagic area of patients with diabetic retinopathy

\subsection{Transformed Color Spaces of the Dust Artifact Area}

Six different colored artificial eyes were photographed five times with a fragment of house dust set on the lens at points P1-P6. We obtained and analyzed a total of 180 images.
Table 1. CngHm in the eight color spaces

\begin{tabular}{ccccc}
\hline Object & RGB & XYZ & CMY & HSL \\
\hline Cng1_hm & $0.044 \pm 0.027$ & $0.047 \pm 0.032$ & $0.044 \pm 0.020$ & $1.2^{\circ} \pm 0.9^{\circ}$ \\
Cng2_hm & $0.067 \pm 0.045$ & $0.056 \pm 0.038$ & $0.027 \pm 0.016$ & $0.013 \pm 0.014$ \\
Cng3_hm & $0.840 \pm 1.186$ & $0.065 \pm 0.042$ & $0.003 \pm 0.003$ & $0.040 \pm 0.025$ \\
\hline Object & HSV & HSI & $\mathbf{L}^{*} \mathbf{a}^{*} \mathbf{b}^{*}$ & $\mathbf{L}^{*} \mathbf{u}^{*} \mathbf{v}^{*}$ \\
\hline Cng1_hm & $1.2^{\circ} \pm 0.9^{\circ}$ & $1.3^{\circ} \pm 1.0^{\circ}$ & $0.024 \pm 0.017$ & $0.024 \pm 0.017$ \\
Cng2_hm & $0.007 \pm 0.008$ & $0.013 \pm 0.013$ & $1.662 \pm 3.284$ & $0.043 \pm 0.045$ \\
Cng3_hm & $0.044 \pm 0.027$ & $0.047 \pm 0.033$ & $0.037 \pm 0.025$ & $0.035 \pm 0.026$ \\
\hline
\end{tabular}

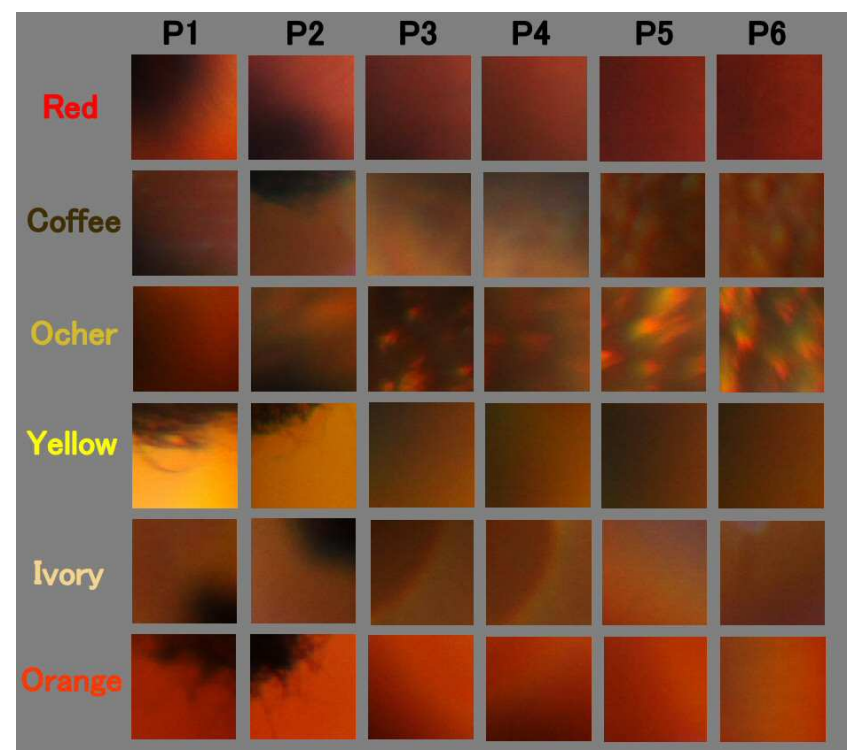

Figure 8. Thirty-six images of the dust artifact clipped from the 180 artificial eye photographs

\subsection{Comparison of the Evaluation of Spaces for House Dust in the Eight Types of Color Spaces}

The color space for an evaluation is Ev. Ev was calculated by substituting aveRGBda and aveRGBpa from equations (7) to (13). Fig. 9 demonstrates the averages and standard deviations of Ev in the eight types of color spaces. In this graph, the bars represent average values, and the lines represent standard deviations. Larger the average of Ev, higher the sensitivity of the color space. Moreover, smaller the standard deviation of Ev in comparison with the average of $\mathrm{Ev}$, better the reproducibility of the color space.

As shown in Fig. 9, the color space with the highest sensitivity and good reproducibility was $\mathrm{L}^{*} \mathrm{v} \mathrm{u}^{*}$ because all these averages were large and all standard deviations were small in comparison with the averages. As the six downward arrows demonstrate, HSV, HSL, HSI, RGB, $\mathrm{XYZ}$, and $\mathrm{L} * \mathrm{a} * \mathrm{~b} *$ each contain an ingredient of low sensitivity. Moreover, as the rectangle shows, CMY contains an ingredient of inferior reproducibility. 

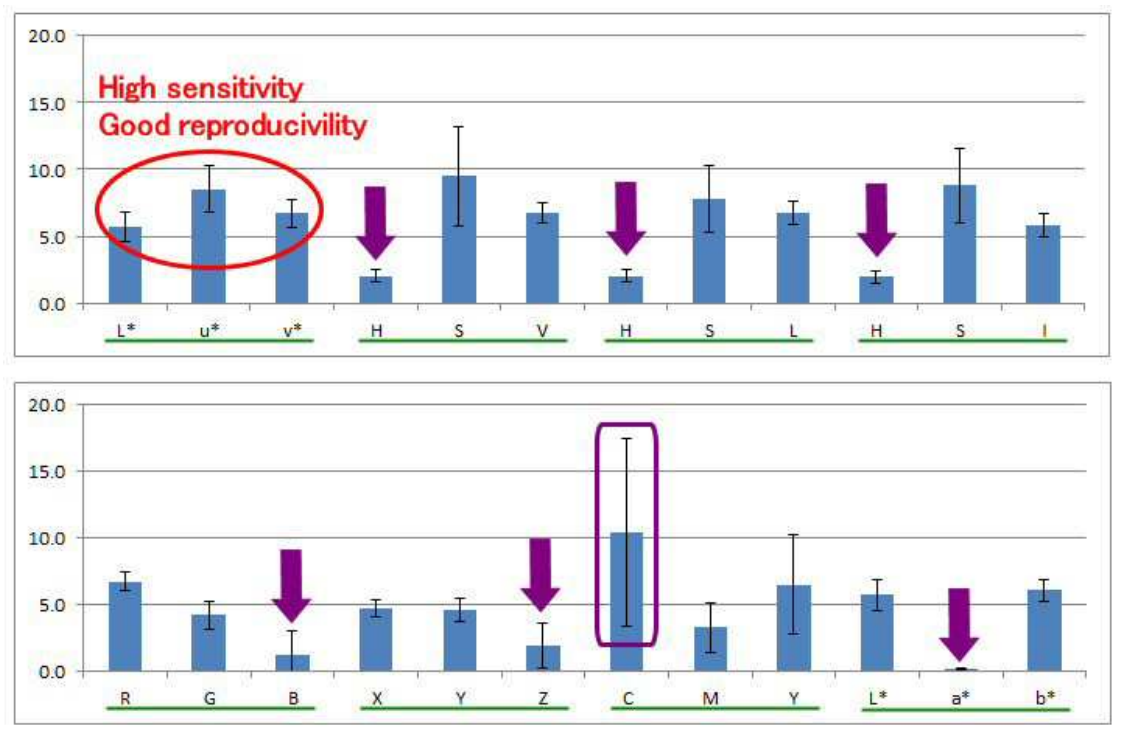

Figure 9. Comparison of eight types of color space

Since these averages were large and these standard deviations were small for value, lightness, and intensity, sensitivity and reproducibility were good. Because all saturation averages were very large, saturation sensitivity was very high. However, since all standard deviations of saturation were not small, reproducibility of saturation was poor. Since all standard deviations and all the averages of hue were small, hue sensitivity was poor.

In " $\mathrm{L}^{*} \mathrm{a} \mathrm{b}^{*}$," because the averages of " $\mathrm{L} *$ " and " $\mathrm{b}$ *" were large, and because the standard deviations of " $\mathrm{L} *$ " and "b*" were small, sensitivity and reproducibility were good. However, since the average of "a*" was very small, the sensitivity of "a*" was poor.

In RGB, because the averages of $\mathrm{R}$ and $\mathrm{G}$ were large, and standard deviations of $R$ and $G$ were small, sensitivity and reproducibility were good. However, since the average of $\mathrm{B}$ was small, the sensitivity of $\mathrm{B}$ was poor.

In $\mathrm{XYZ}$, because the averages of $\mathrm{X}$ and $\mathrm{Y}$ were marginally large, and standard deviations of $\mathrm{X}$ and $\mathrm{Y}$ were small, sensitivity and reproducibility were good. However, since the average of $Z$ was small, the sensitivity of $Z$ was poor.
In CMY, because the averages were large, the sensitivity of CMY was very high. However, because these standard deviations were not small, the reproducibility of CMY was poor.

\subsection{Analysis of $L^{*} u^{*} v^{*}$ Color Space}

Fig. 10: The averages and standard deviations of $\mathrm{L}^{*}$.

In these graphs, the bars represent average values, and the lines represent standard deviations. The bars are colored red, coffee, ocher, yellow, ivory, and orange from left to right. Table 2 shows the averages and standard deviations of L*. Points P1 and P2 are on an object lens. Points P3 and P4 are on a photographic optical system. Points P5 and P6 are on an illumination optical system.

If the averages are $>1.0$, the ingredient can distinguish small hemorrhages from dust artifacts. Moreover, if standard deviations are small in comparison with the averages, reproducibility is good. As shown in Fig. 10, the house dust position "on an object lens" had the highest sensitivity and good reproducibility.

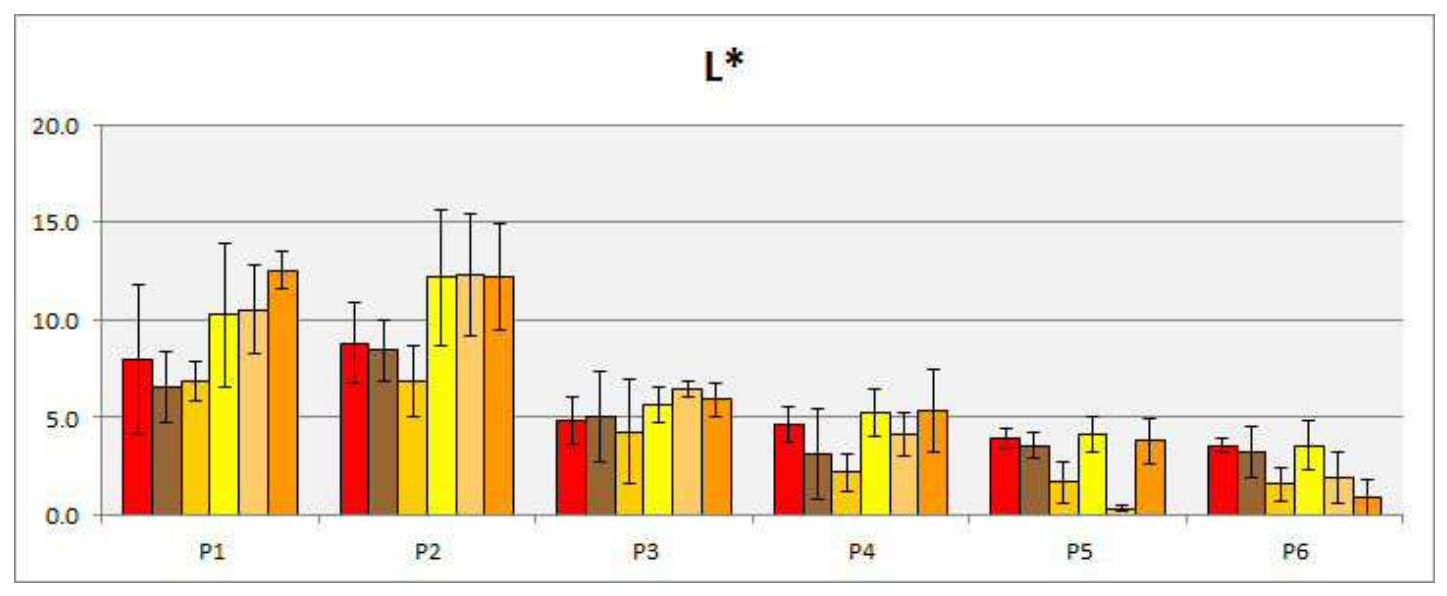

Figure 10. The averages and standard deviations of $L^{*}$ 
Table 2. The averages and standard deviations of $L^{*}$

\begin{tabular}{ccccccc}
\hline & Red & Coffee & Ocher & Yellow & Ivory & Orange \\
\hline P1 & $7.92 \pm 3.83$ & $6.52 \pm 1.82$ & $6.86 \pm 1.01$ & $10.24 \pm 3.72$ & $10.54 \pm 2.25$ & $12.51 \pm 0.96$ \\
P2 & $8.82 \pm 2.06$ & $8.46 \pm 1.58$ & $6.82 \pm 1.81$ & $12.16 \pm 3.50$ & $12.27 \pm 3.13$ & $12.20 \pm 2.75$ \\
P3 & $4.85 \pm 1.19$ & $5.07 \pm 2.34$ & $4.28 \pm 2.71$ & $5.66 \pm 0.94$ & $6.44 \pm 0.37$ & $5.91 \pm 0.87$ \\
P4 & $4.62 \pm 0.90$ & $3.12 \pm 2.31$ & $2.18 \pm 0.92$ & $5.25 \pm 1.20$ & $4.15 \pm 1.08$ & $5.35 \pm 2.13$ \\
P5 & $3.92 \pm 0.48$ & $3.58 \pm 0.69$ & $1.68 \pm 1.07$ & $4.12 \pm 0.88$ & $0.35 \pm 0.16$ & $3.80 \pm 1.18$ \\
P6 & $3.55 \pm 0.34$ & $3.22 \pm 1.34$ & $1.58 \pm 0.82$ & $3.58 \pm 1.24$ & $1.93 \pm 1.35$ & $0.89 \pm 0.90$ \\
\hline
\end{tabular}

As shown in Table 2, the house dust position "on a photographic optical system" had high sensitivity because the average values were $>2.18$. Moreover, because standard deviations were small in comparison with the averages, the house dust position "on a photographic optical system" had good reproducibility, and because the average values were $>1.58$, the house dust position "on an illumination optical system" had good sensitivity except for the combinations of "P5 and ivory" and "P6 and orange."
Moreover, since the standard deviations were small in comparison with the averages, the house dust position "on an illumination optical system" had good reproducibility except for the combinations of "P5 and ivory" and "P6 and orange."

Fig. 11 and Table 3 show the averages and standard deviations of $\mathrm{u}^{*}$.

As shown in Fig. 11, the house dust position "on an object lens" had good sensitivity and reproducibility.

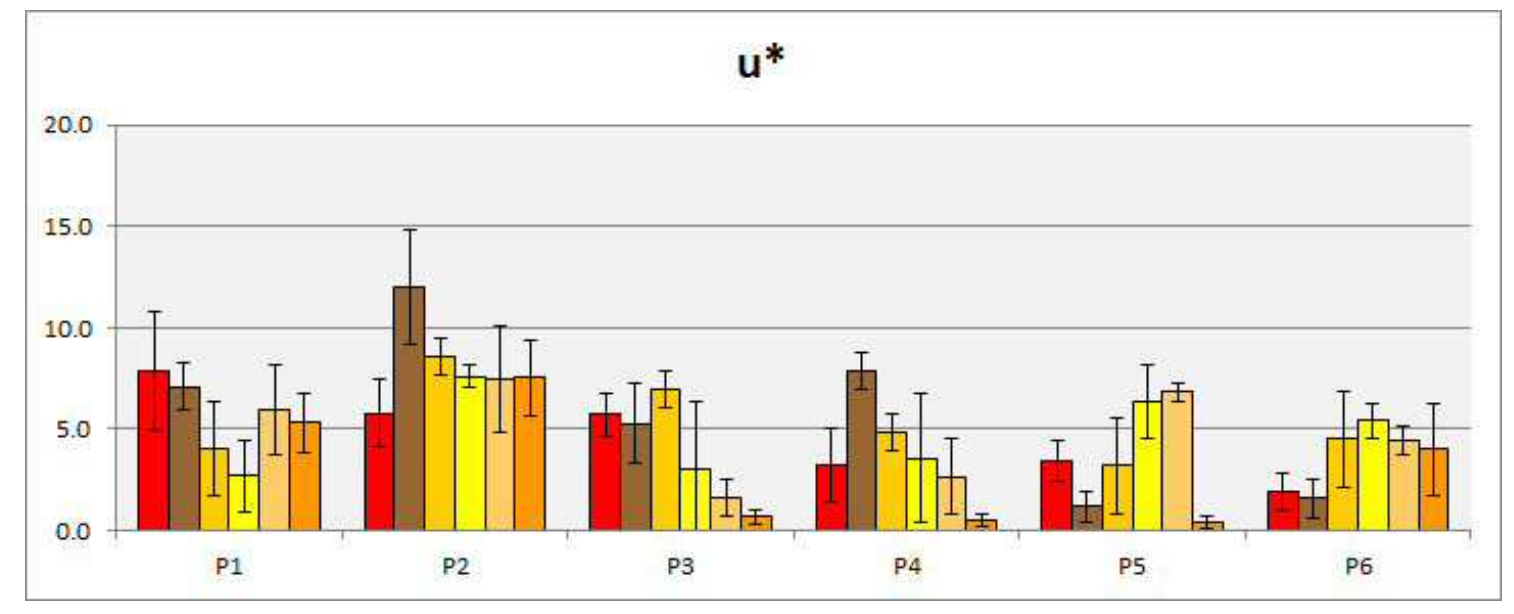

Figure 11. The averages and standard deviations of $u^{*}$

Table 3. The averages and standard deviations of $u *$

\begin{tabular}{ccccccc}
\hline & Red & Coffee & Ocher & Yellow & Orange & \\
\hline P1 & $7.90 \pm 2.93$ & $7.08 \pm 1.14$ & $4.08 \pm 2.31$ & $2.70 \pm 1.74$ & $5.94 \pm 2.21$ \\
P2 & $5.80 \pm 1.66$ & $12.00 \pm 2.83$ & $8.61 \pm 0.90$ & $7.60 \pm 0.55$ & $7.50 \pm 2.62$ \\
P3 & $5.72 \pm 1.04$ & $5.28 \pm 1.94$ & $6.95 \pm 0.95$ & $3.01 \pm 3.37$ & $1.62 \pm 0.94$ \\
P4 & $3.24 \pm 1.81$ & $7.85 \pm 0.90$ & $4.82 \pm 0.89$ & $3.57 \pm 3.15$ & $2.66 \pm 1.89$ \\
P5 & $3.46 \pm 0.99$ & $1.20 \pm 0.78$ & $3.20 \pm 2.40$ & $6.37 \pm 1.82$ & $6.81 \pm 0.46$ \\
P6 & $1.94 \pm 0.88$ & $1.60 \pm 0.94$ & $4.51 \pm 2.34$ & $5.43 \pm 0.85$ & $0.50 \pm 0.28$ & $4.45 \pm 0.71$ \\
\hline
\end{tabular}

As shown in Table 3, the house dust position "on a photographic optical system" had high sensitivity because the average values were $>1.62$ except for the combinations of "P3 and orange" and "P4 and orange." Moreover, since the standard deviations were small in comparison with the averages, the house dust position "on a photographic optical system" had good reproducibility except for the combinations of "P3 and yellow," "P4 and yellow," "P3 and orange," and "P4 and orange." Because the average values were $>1.20$, the house dust position "on an illumination optical system" had good sensitivity except for the combination of "P5 and orange." Moreover, since the standard deviations were small in comparison with the averages, the house dust position "on an illumination 
optical system" had good reproducibility except for the combination of "P5 and orange."

Fig. 12 and Table 4 show the averages and standard deviations of $\mathrm{v}^{*}$.
As shown in Fig. 12, the house dust position "on an object lens" had very high sensitivity and good reproducibility.

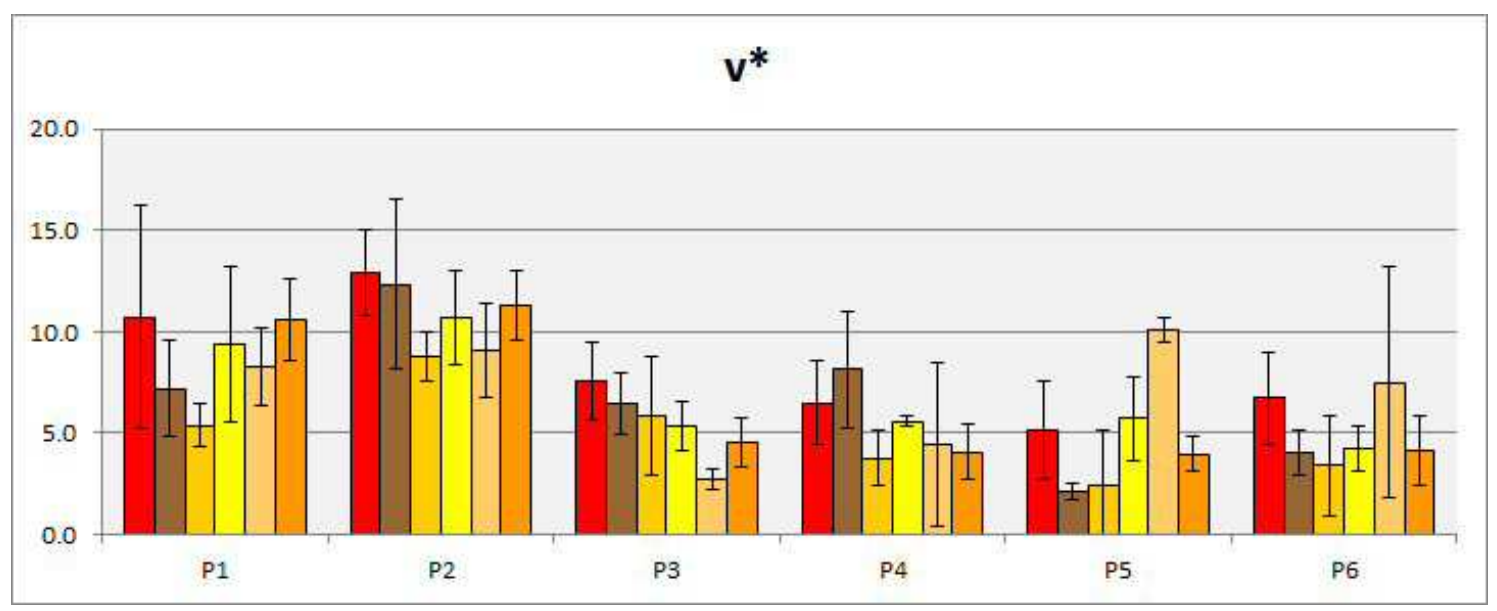

Figure 12. The averages and standard deviations of $v^{*}$

Table 4. The averages and standard deviations of $v^{*}$

\begin{tabular}{ccccccc}
\hline & Red & Coffee & Ocher & Yellow & Orange & Ivory \\
\hline P1 & $10.73 \pm 5.52$ & $7.21 \pm 2.38$ & $5.38 \pm 1.03$ & $9.35 \pm 3.84$ & $8.30 \pm 1.92$ \\
P2 & $12.92 \pm 2.10$ & $12.32 \pm 4.18$ & $8.80 \pm 1.19$ & $10.68 \pm 2.32$ & $9.10 \pm 2.34$ \\
P3 & $7.54 \pm 1.92$ & $6.47 \pm 1.52$ & $5.84 \pm 2.94$ & $5.34 \pm 1.22$ & $2.74 \pm 0.48$ \\
P4 & $6.47 \pm 2.06$ & $8.14 \pm 2.86$ & $3.79 \pm 1.37$ & $5.56 \pm 0.25$ & $4.45 \pm 4.01$ \\
P5 & $5.17 \pm 2.43$ & $2.12 \pm 0.41$ & $2.47 \pm 2.64$ & $5.71 \pm 2.06$ & 4.03 & $10.07 \pm 0.62$ \\
P6 & $6.72 \pm 2.23$ & $4.04 \pm 1.08$ & $3.41 \pm 2.45$ & $4.27 \pm 1.09$ & $3.95 \pm 0.86$ & $7.50 \pm 5.67$ \\
\hline
\end{tabular}

As shown in Table 4, the house dust position "on a photographic optical system" had high sensitivity because the average values were $>2.74$. Moreover, since the standard deviations were small in comparison with the averages, the house dust position "on a photographic optical system" had good reproducibility except for the combination of "P4 and ivory." Because the average values were $>2.12$, the house dust position "on an illumination optical system" had good sensitivity. Moreover, since the standard deviations were small in comparison with the averages, the house dust position "on a illumination optical system" had good reproducibility except for the combinations of "P5 and ocher" and "P6 and ivory."

\section{Discussions}

Fundus photographs were taken in five patients with diabetic retinopathy. We constructed an experimental device and six artificial eyes. The experimental device was composed of an illumination optical system and a photographic optical system similar to that of a fundus camera. The fundi of the artificial eyes were painted in six colors: red, coffee, ocher, yellow, ivory, and orange. House dust particles on the object lens and the two other lenses were photographed under each artificial eye. The RGB color space was measured in the following four areas: the hemorrhagic area, the perihemorrhagic area, the dust artifact, and the periartifact area. The RGB color space was transformed into seven types of color spaces: XYZ, CMY, HSL, HSV, HSI, L*a*b*, and $\mathrm{L}^{*} \mathrm{u}^{*} \mathrm{v}^{*}$. We compared eight types of color spaces using the evaluation space "Ev."

The optimal color space was $\mathrm{L}^{*} \mathrm{u}^{*} \mathrm{v}^{*}$ because it showed the highest sensitivity and the best reproducibility. However, HSV, HSL, HSI, RGB, XYZ, and L*a*b* each had an ingredient of low sensitivity. The ingredients of low sensitivity were the colors blue, "Z", and hue. Moreover, CMY consisted of cyan, which is a mixture of blue and green, an ingredient of bad reproducibility. The ingredient " $Z$ " expresses the degree of blueness. The present experiment demonstrated that the ingredients related to blue had neither high sensitivity nor good reproducibility. In addition, hue was of low sensitivity. The results from this experiment demonstrated that dust artifacts did not result in transformation of colors. In contrast, saturation had high sensitivity and good reproducibility, and dust artifacts create a spotty pattern. The concentration of color is one of the important elements in the detection of dust artifacts. The results from this experiments demonstrated 
that saturation, the ingredient that shows the concentration of color, can distinguish small hemorrhages from dust artifacts. Furthermore, "L*," value, lightness, and intensity had high sensitivity and good reproducibility. Dust artifacts are marginally dark because house dust particles interrupt light. In order to distinguish small hemorrhages from dust artifacts, this experimental result theoretically demonstrated that the ingredients of "brightness" had high sensitivity and good reproducibility.

Next, we analyzed the $\mathrm{L}^{*} \mathrm{u} * \mathrm{v} *$ color space and compared the following three types of house dust positions: "on an object lens," "on a photographic optical system," and "on an illumination optical system." With regard to " $L *$," the position "on an object lens" had the highest sensitivity and the best reproducibility. The position 'on a photographic optical system' had good sensitivity and reproducibility. However, the position "on an illumination optical system" had good sensitivity and reproducibility only under certain conditions. The house dust position "on an object lens" had the highest sensitivity and the best reproducibility. With regard to " $u *$," the position "on an object lens" had the highest sensitivity and the best reproducibility. However, the positions "on a photographic optical system" and "on an illumination optical system" had good sensitivity and reproducibility only under certain conditions. With regard to " $\mathrm{v} *$," the position "on an object lens" had the highest sensitivity and the best reproducibility, whereas the position "on a photographic optical system" had high sensitivity and good reproducibility. However, the position "on an illumination optical system" had high sensitivity and good reproducibility only under certain conditions. Finally we experimented with the artificial eyes having six types of fundus colors. No differences were found among the six types of colors.

\section{Conclusions}

1. The $\mathrm{L}^{*} \mathrm{u}^{*} \mathrm{v}^{*}$ color space was the optimal one, which demonstrated the highest sensitivity and the best reproducibility. This result demonstrated that the " $\mathrm{L}^{*} \mathrm{u}^{*} \mathrm{v} *$ " color space could distinguish small hemorrhages from dust artifacts.

2. The house dust position "on an object lens" had the highest sensitivity and the best reproducibility. However, the positions "on a photographic optical system" and "on an illumination optical system" had high sensitivity and good reproducibility only under certain conditions.

3. Saturation had high sensitivity and good reproducibility. The ingredients of "brightness," which included "L*," value, lightness, and intensity had high sensitivity and good reproducibility. However, the ingredients related to blue had neither high sensitivity nor good reproducibility. Moreover, dust artifacts did not cause change of hue.

4. No differences were found among the six types of fundus colors.

\section{Acknowledgements}

Photographs from diabetic retinopathy patients were provided by Hiroshima University Hospital. For research expenses, the intramural budget of Hiroshima International University was used. We are very thankful to all those who helped in our research.

\section{References}

[1] Z.T. Bloomgarden, "American Diabetes Association Annual Meeting, 1999, Diabetes and obesity”, Diabetes Care 2000, Vol.23, No.1, pp118-124.

[2] T. Tsuchiya, "Measure Against Lifestyle Related Disease", JMAJ 2006, Vol.49 No.3, pp132-134.

[3] S.S. Savant, H.B. Chandalia, "Diabetic retinopathy", Intnl. J. Dev. Contries 1991, Vol.10, pp9-25.

[4] American Diabetes Association, Diabetic retinopathy, Clinical Diabetes 2001, Vol.19 No.1, pp29-32.

[5] J.B. Brown, K.L. Pedula, K.H. Summers, "Diabetic retinopathy", Diabetes Care 2003, Vol.26 No.9, pp2637-2642.

[6] E. Duh, "Diabetic Retinopathy”, Humana Press, 2008.

[7] S. Garg, R.M. Davis, "Diabetic retinopathy screening update", Clinical diabetes 2009, Vol.27 No.4, pp140-145.

[8] I.U. Scott, N.M. Bressler, S.B. Bresseler, et al., "Agreement between clinical and reading center gradings of diabetic retinopathy severity level at baseline in a phase 2 study of intravitreal bevacizumab for diabetic macular edema", Retina 2008, Vol. 28 No.1, pp36-40.

[9] M.S. Figueroa, I. Contreras, S. Noval, "Anti-angiogetic drugs as an adjunctive therapy in the surgical treatment of diabetic retinopathy", Curr Diabetes Rev 2009, Vol.5 No.1, pp52-56.

[10] E. Fernández-Caldas, W.L. Trudeau, D.K. Ledford, "Environmental control of indoor biologic agents", J Allergy Clin Immunol 1994, Vol.2 No.2, pp404-412.

[11] A.G. Oomen, J.P.C.M. Janssen, A. Dusseldorp, et al., "Ecposure to chemicals via house dust", RIVM Report 609021064/2008, pp11-18.

[12] M. Born, E. Wolf, Principles of Optics $7^{\text {th }}$ expanded edition, Electromagnetic Theory of Propagation, Interference and Diffraction of Light, Cambridge Univ. Press; 1999.

[13] R.G. Willson, M.W. Maimone, A.E. Johnson, et al., "An Optical Model for Image Artifacts Produced by Dust Particles on Lenses", Proceedings of ISAIRAS 2005 Conference, 2005.

[14] A.V. Shukla, Clinical Optics Primer for Ophthalmic Medical Personnel, A Guide to Laws, Formulae, Calculations, and Clinical Applications, SLACK Inc; 2009.

[15] D.D. David, R. Prescott, S. Kennedy, "Simultaneous stereoscopic fundus camera incorporating a single optical axis", Invest. Ophthalmol. Vis. Sci. March, 1980, pp289-297.

[16] R. Zeimer, S. Zou, T. Meeder, et al., "A fundus camera dedicated to the screening of diabetic retinopathy in the 
primary-care physician's office", IOVS May 2002, Vol. 43 No.5, pp1581-1587.

[17] R.F. Lyon, "Prism-Based Color Separation for Professional Digital Photography,", Proceedings of IS\&T's PICS 2000 Conference: Image Processing, Image Quality, Image Capture Systems Conference, 2000, Oregon, USA.

[18] A.E. Dirik, H.T. Sencar, N. Memon, "Source Camera Identification Based on Sensor dust Characteristics", The proceedings of Signal Processing Applications for Public Security and Forensics 2007, SAFE'07, IEEE Workshop 2007.

[19] A. Zamfir, A. Drimbarean, M. Zamfir, et al., "An Optic Model of the Appearance of Blemishes in Digital Photographs", Proceedings of SPIE 2007, Vol. 6502, Digital Photography 3 .

[20] R. Bergman, R. Maurer, H. Nachlieli, et al., "Comprehensive Solutions for Removal of Dust and Scratches from Images", Journal of Electronic Imaging 2008, Vol.17 No.1, pp1-25.

[21] A.E. Dirik, H.T. Sencar, N. Memon, "Digital Single Lens Reflex Camera Identification From Traces of Sensor Dust", IEEE Trans Info For and Sec 2008, Vol.3 No.3, pp539-552.

[22] P.J. Saine, "Errors in Fundus Photography", Journal of Ophthalmic Photography 1984, Vol.7 No.2, pp120-122.

[23] H. Narasimha-Iyer, A. Can, B. Roysam, et al., "Robust Detection and Classification of Longitudinal Changes in Color Retinal Fundus Images for Monitoring Diabetic Retinopathy”, IEEE Trans Biomed Eng 2006, Vol.53 No.6, pp1084-1098.

[24] H. Narasima-lyer, A. Can, B. Roysam, et al., "Integrated analysis of vascular and nonvascular changes from color retinal fundus image sequences", IEEE Trans Biomed Eng 2007, Vol.54 No.8, pp1436-1445.

[25] J. Koenderink, Color for the sciences, The MIT Press; 2009.

[26] N. Suzuki, "Basic Research for Distinguishing Small Retinal Hemorrhages from Dust Artifact by using Hue, Lightness, and Saturation Color Space", World Academy of Science, Engineering and Technology 2012, Vol.65, pp 844-852.

[27] B. Jahne, Practical Handbook on Image Processing for Scientific Applications, CRC Press, 1997.

[28] B.G. Haskell, A. Puri, A.N. Netravali, Digital Video: An Introduction to MPEG-2, Chapman \& Hall, 1997.

[29] A. Hanbury, J. Serra, "Mathematical Morphology in the HLS Color Space", Proceeding of 12th British Machine Vision Conference, 2001, Manchester, UK.

[30] M. Tkalcic, J.F. Tasic, Color spaces - perceptual, historical and applicational background, EUROCON 2003, Computer as a tool, The IEEE Region 8, Vol.1, pp304-308.

[31] A. Ford, A. Roberts, Color space conversion, Westminster University, London, 1998.

[32] Jasc Software, Inc., Chapter 8: Making color and Tonal Corrections, Paint Shop Pro 8 User Guide, 2002.

[33] R.A. Peters II, "Mathematical morphology for angle-valued images", Proceedings of the SPIE, Nonlinear Image Processing VIII 3026, 1997.

[34] Nikon Corporations. Nikon D1X Operators Manual.

[35] Canon Inc. Canon EOS 50D Instruction Manual, 2008. 